\title{
Strong convergence theorems for equilibrium problems and fixed point problem of multivalued nonexpansive mappings via hybrid projection method
}

\author{
Ali Abkar ${ }^{1}$ and Mohammad Eslamian²*
}

\section{${ }^{*}$ Correspondence:}

mhmdeslamian@gmail.com

${ }^{2}$ Young Researchers Club, Babol

Branch, Islamic Azad University,

Babol, Iran

Full list of author information is

available at the end of the article

\begin{abstract}
In this paper, a new iterative process by the hybrid projection method is constructed. Strong convergence of the iterative process to a common element of the set of common fixed points of a finite family of generalized nonexpansive multivalued mappings and the solution set of two equilibrium problems in a Hilbert space is proved. Our results extend some important recent results.

MSC: $47 \mathrm{H} 10 ; 47 \mathrm{H} 09$
\end{abstract}

Keywords: equilibrium problem; hybrid projection method; strong convergence; common fixed point; generalized nonexpansive multivalued mapping

\section{Introduction}

Let $C$ be a nonempty closed convex subset of a Hilbert space $H$. A subset $C \subset H$ is called proximal if for each $x \in H$ there exists an element $y \in C$ such that

$$
\|x-y\|=\operatorname{dist}(x, C)=\inf \{\|x-z\|: z \in C\} .
$$

We denote by $C B(C)$ and $P(C)$ the collection of all nonempty closed bounded subsets and nonempty proximal bounded subsets of $C$, respectively. The Hausdorff metric $H$ on $C B(H)$ is defined by

$$
H(A, B):=\max \left\{\sup _{x \in A} \operatorname{dist}(x, B), \sup _{y \in B} \operatorname{dist}(y, A)\right\},
$$

for all $A, B \in C B(H)$.

Let $T: H \rightarrow 2^{H}$ be a multivalued mapping. An element $x \in H$ is said to be a fixed point of $T$, if $x \in T x$. The set of fixed points of $T$ will be denoted by $F(T)$.

Definition 1.1 A multivalued mapping $T: H \rightarrow C B(H)$ is called

(i) nonexpansive if

$$
H(T x, T y) \leq\|x-y\|, \quad x, y \in H .
$$


(ii) quasi-nonexpansive if $F(T) \neq \emptyset$ and $H(T x, T p) \leq\|x-p\|$ for all $x \in H$ and all $p \in F(T)$.

Recently, J. Garcia-Falset, E. Llorens-Fuster and T. Suzuki [1] introduced a new condition on singlevalued mappings, called condition $(E)$, which is weaker than nonexpansiveness.

Definition 1.2 A mapping $T: H \rightarrow H$ is said to satisfy the condition $\left(E_{\mu}\right)$ provided that

$$
\|x-T y\| \leq \mu\|x-T x\|+\|x-y\|, \quad x, y \in H .
$$

We say that $T$ satisfies the condition (E) whenever $T$ satisfies $\left(E_{\mu}\right)$ for some $\mu \geq 1$.

Now we modify this condition for multivalued mappings as follows (see also [2]):

Definition 1.3 A multivalued mapping $T: H \rightarrow C B(H)$ is said to satisfy the condition $(P)$ provided that

$$
H(T x, T y) \leq \mu \operatorname{dist}(x, T x)+\eta\|x-y\|, \quad x, y \in H,
$$

for some $\mu, \eta \geq 1$.

It is obvious that every nonexpansive multivalued mapping satisfies the condition $(P)$. The theory of multivalued mappings has applications in control theory, convex optimization, differential equations and economics. Theory of nonexpansive multivalued mappings is harder than the corresponding theory of nonexpansive single valued mappings. Different iterative processes have been used to approximate fixed points of multivalued nonexpansive mappings (see [3-8]). Let $\Phi$ be a bifunction from $C \times C$ into $\mathbb{R}$, where $\mathbb{R}$ is the set of real numbers. The equilibrium problem for $\Phi: C \times C \rightarrow \mathbb{R}$ is to find $x \in C$ such that

$$
\Phi(x, y) \geq 0, \quad \forall y \in C
$$

The set of solutions is denoted by $E P(\Phi)$. It is well known that this problem is closely related to minimax inequalities (see [9] and [10]). The equilibrium problem includes fixed point problems, optimization problems and variational inequality problems as special cases. Some methods have been proposed to solve the equilibrium problem, see, for example, [11-14].

Recently, many authors have studied the problems of finding a common element of the set of fixed points of nonexpansive single valued mappings and the set of solutions of an equilibrium problem in the framework of Hilbert spaces: see, for instance, [15-29] and the references therein. In this paper, a new iterative process by the hybrid projection method is constructed. Strong convergence of the iterative process to a common element of a set of common fixed points of a finite family of multivalued mappings satisfying the condition (P) and the solution set of two equilibrium problems in a Hilbert space is proved. Our results generalize some results of Tada, Takahashi [15] and many others. 


\section{Preliminaries}

Let us recall the following definitions and results which will be used in the sequel.

Lemma 2.1 ([6]) Let $H$ be a real Hilbert space. Then for $i, j=1,2, \ldots, k$ we have

$$
\left\|a_{1} x_{1}+a_{2} x_{2}+\cdots+a_{k} x_{k}\right\|^{2} \leq a_{1}\left\|x_{1}\right\|^{2}+a_{2}\left\|x_{2}\right\|^{2}+\cdots+a_{k}\left\|x_{k}\right\|^{2}-a_{i} a_{j}\left\|x_{i}-x_{j}\right\|^{2}
$$

for all $x_{i}, x_{j} \in H$ and $a_{i}, a_{j} \in[0,1]$ with $\sum_{i=1}^{k} a_{i}=1$.

Let $C$ be a closed convex subset of $H$. For every point $x \in H$, there exists a unique nearest point in $C$, denoted by $P_{C} x$ such that

$$
\left\|x-P_{C} x\right\| \leq\|x-y\|, \quad \forall y \in C .
$$

$P_{C}$ is called the metric projection of $H$ onto $C$. It is well known that $P_{C}$ is a nonexpansive mapping.

Lemma 2.2 ([16]) Let $C$ be a closed convex subset of $H$. Given $x \in H$ and a point $z \in C$. Then $z=P_{C} x$ if and only if

$$
\langle x-z, z-y\rangle \geq 0, \quad \forall y \in C
$$

Lemma 2.3 ([30]) Let $C$ be a closed convex subset of $H$. Then for all $x \in H$ and $y \in C$ we have

$$
\left\|y-P_{C} x\right\|^{2}+\left\|x-P_{C} x\right\|^{2} \leq\|x-y\|^{2} .
$$

For solving the equilibrium problem, we assume that the bifunction $\Phi$ satisfies the following conditions:

(A1) $\Phi(x, x)=0$ for any $x \in C$,

(A2) $\Phi$ is monotone, i.e., $\Phi(x, y)+\Phi(y, x) \leq 0$ for any $x, y \in C$,

(A3) $\Phi$ is upper-hemicontinuous, i.e., for each $x, y, z \in C$,

$$
\limsup _{t \rightarrow 0^{+}} \Phi(t z+(1-t) x, y) \leq \Phi(x, y)
$$

(A4) $\Phi(x, \cdot)$ is convex and lower semicontinuous for each $x \in C$.

The following lemma was proved in [11].

Lemma 2.4 Let $C$ be a nonempty closed convex subset of $H$ and let $\Phi$ be a bifunction of $C \times C$ into $\mathbb{R}$ satisfying (A1)-(A4). Let $r>0$ and $x \in H$. Then, there exists $z \in C$ such that

$$
\Phi(z, y)+\frac{1}{r}\langle y-z, z-x\rangle \geq 0 \quad \forall y \in C .
$$

The following lemma was given in [14]. 
Lemma 2.5 Assume that $\Phi: C \times C \rightarrow \mathbb{R}$ satisfies (A1)-(A4). For $r>0$ and $x \in H$, define a mapping $T_{r}: H \rightarrow C$ as follows:

$$
T_{r} x=\left\{z \in C: \Phi(z, y)+\frac{1}{r}\langle y-z, z-x\rangle \geq 0, \forall y \in C\right\} .
$$

Then, the following hold:

(i) $T_{r}$ is single valued;

(ii) $T_{r}$ is firmly nonexpansive, i.e., for any $x, y \in H$,

$$
\left\|T_{r} x-T_{r} y\right\|^{2} \leq\left\langle T_{r} x-T_{r} y, x-y\right\rangle
$$

(iii) $F\left(T_{r}\right)=E P(\Phi)$;

(iv) $E P(\Phi)$ is closed and convex.

The following lemma was proved in [31] for nonexpansive multivalued mappings. The statement is true for quasi-nonexpansive multivalued mappings as well. To avoid repetition, we omit the details of the proof.

Lemma 2.6 Let $C$ be a closed convex subset of a real Hilbert space H. Let $T: C \rightarrow C B(C)$ be a quasi-nonexpansive multivalued mapping such that $T(p)=\{p\}$ for all $p \in F(T)$. Then $F(T)$ is closed and convex.

Now, following Shahzad and Zegeye [3], we remove the restriction $T(p)=\{p\}$ for all $p \in F(T)$. Let $T: C \rightarrow P(C)$ be a multivalued mapping and

$$
P_{T}(x)=\{y \in T x:\|x-y\|=\operatorname{dist}(x, T x)\} .
$$

We use a similar argument as in the proof of Lemma 3.1 in [31] to obtain the following lemma.

Lemma 2.7 Let $C$ be a closed convex subset of a real Hilbert space $H$. Let $T: C \rightarrow P(C)$ be a multivalued mapping such that $P_{T}$ is quasi-nonexpansive. Then $F(T)$ is closed and convex.

Note that for all $p \in F(T), P_{T}(p)=\{p\}$. We remark that there exist some examples of multivalued mappings for which $P_{T}$ is nonexpansive (see [3] for details), so that the assumption on $T$ is not artificial.

\section{The main result}

Theorem 3.1 Let $C$ be a nonempty closed convex subset of a real Hilbert space $H, \Phi_{1}$ and $\Phi_{2}$ be two bifunctions of $C \times C$ into $\mathbb{R}$ satisfying (A1)-(A4). Let $T_{i}: C \rightarrow C B(C),(i=$ $1,2, \ldots, m)$, be a finite family of quasi-nonexpansive multivalued mappings, each satisfying the condition $(P)$. Assume further that $\mathcal{F}=\bigcap_{i=1}^{m} F\left(T_{i}\right) \cap E P\left(\Phi_{1}\right) \cap E P\left(\Phi_{2}\right) \neq \emptyset$ and $T_{i}(p)=$ $\{p\},(i=1,2, \ldots, m)$, for each $p \in \mathcal{F}$. For $C_{0}=C$, let $\left\{x_{n}\right\}$ and $\left\{u_{n}\right\}$ be sequences generated by 
the following algorithm:

$$
\left\{\begin{array}{l}
x_{0} \in C, \\
u_{n} \in C \text { such that } \Phi_{1}\left(u_{n}, y\right)+\frac{1}{r_{n}}\left\langle y-u_{n}, u_{n}-x_{n}\right\rangle \geq 0 ; \quad \forall y \in C, \\
u_{n}^{\prime} \in C \text { such that } \Phi_{2}\left(u_{n}^{\prime}, y\right)+\frac{1}{s_{n}}\left\langle y-u_{n}^{\prime}, u_{n}^{\prime}-x_{n}\right\rangle \geq 0 ; \quad \forall y \in C, \\
v_{n}=\delta_{n} u_{n}+\left(1-\delta_{n}\right) u_{n}^{\prime}, \\
w_{n}=a_{n, 0} v_{n}+a_{n, 1} z_{n, 1}+\cdots+a_{n, m} z_{n, m}, \\
y_{n}=b_{n, 0} v_{n}+b_{n, 1} z_{n, 1}^{\prime}+\cdots+b_{n, m} z_{n, m}^{\prime}, \\
C_{n+1}=\left\{v \in C_{n}:\left\|y_{n}-v\right\| \leq\left\|x_{n}-v\right\|\right\}, \\
x_{n+1}=P_{C_{n+1}} x_{0}: \quad \forall n \geq 0,
\end{array}\right.
$$

where $z_{n, i} \in T_{i} v_{n}, z_{n, i}^{\prime} \in T_{i} w_{n}$ for $i=1,2, \ldots, m$. Assume that $\left\{a_{n, j}\right\},\left\{b_{n, j}\right\},\left\{\delta_{n}\right\}$ and $\left\{r_{n}\right\},\left\{s_{n}\right\}$ satisfy the following conditions:

(i) $\left\{a_{n, j}\right\},\left\{b_{n, j}\right\},\left\{\delta_{n}\right\} \subset[a, b] \subset(0,1)(j=0,1,2, \ldots, m)$,

(ii) $\left\{r_{n}\right\},\left\{s_{n}\right\} \subset(0, \infty)$, and $\liminf _{n \rightarrow \infty} r_{n}>0$ and $\liminf _{n \rightarrow \infty} s_{n}>0$.

Then, the sequences $\left\{x_{n}\right\}$ and $\left\{v_{n}\right\}$ converge strongly to $P_{\mathcal{F}} x_{0}$.

Proof First, we show that $\mathcal{F} \subset C_{n}$ for all $n \geq 0$. Fix $q \in \mathcal{F}$. We set

$$
T_{r}^{\Phi_{1}}(x)=\left\{z \in C: \Phi_{1}(z, y)+\frac{1}{r}\langle y-z, z-x\rangle \geq 0 \forall y \in C\right\} .
$$

Hence we have $u_{n}=T_{r_{n}}^{\Phi_{1}} x_{n}$ and $u_{n}^{\prime}=T_{s_{n}}^{\Phi_{2}} x_{n}$. By Lemma 2.5, we have

$$
\left\|u_{n}-q\right\|=\left\|T_{r_{n}}^{\Phi_{1}} x_{n}-T_{r_{n}}^{\Phi_{1}} q\right\| \leq\left\|x_{n}-q\right\|
$$

and

$$
\left\|u_{n}^{\prime}-q\right\|=\left\|T_{s_{n}}^{\Phi_{2}} x_{n}-T_{s_{n}}^{\Phi_{2}} q\right\| \leq\left\|x_{n}-q\right\|,
$$

which implies that

$$
\left\|v_{n}-q\right\| \leq \delta_{n}\left\|u_{n}-q\right\|+\left(1-\delta_{n}\right)\left\|u_{n}^{\prime}-q\right\| \leq\left\|x_{n}-q\right\|
$$

Since $T_{i}$ is quasi-nonexpansive, for $i=1,2, \ldots, m$, we have

$$
\begin{aligned}
\left\|w_{n}-q\right\| & =\left\|a_{n, 0} v_{n}+a_{n, 1} z_{n, 1}+\cdots+a_{n, m} z_{n, m}-q\right\| \\
& \leq a_{n, 0}\left\|v_{n}-q\right\|+a_{n, 1}\left\|z_{n, 1}-q\right\|+\cdots+a_{n, m}\left\|z_{n, m}-q\right\| \\
& \leq a_{n, 0}\left\|x_{n}-q\right\|+a_{n, 1} \operatorname{dist}\left(z_{n, 1}, T_{1} q\right)+\cdots+a_{n, m} \operatorname{dist}\left(z_{n, m}, T_{m} q\right) \\
& \leq a_{n, 0}\left\|x_{n}-q\right\|+a_{n, 1} H\left(T_{1} v_{n}, T_{1} q\right)+\cdots+a_{n, m} H\left(T_{m} v_{n}, T_{m} q\right) \\
& \leq a_{n, 0}\left\|x_{n}-q\right\|+a_{n, 1}\left\|v_{n}-q\right\|+\cdots+a_{n, m}\left\|v_{n}-q\right\| \\
& \leq a_{n, 0}\left\|x_{n}-q\right\|+a_{n, 1}\left\|x_{n}-q\right\|+\cdots+a_{n, m}\left\|x_{n}-q\right\| \leq\left\|x_{n}-q\right\|,
\end{aligned}
$$


and also

$$
\begin{aligned}
\left\|y_{n}-q\right\| & =\left\|b_{n, 0} v_{n}+b_{n, 1} z_{n, 1}^{\prime}+\cdots+b_{n, m} z_{n, m}^{\prime}-q\right\| \\
& \leq b_{n, 0}\left\|v_{n}-q\right\|+b_{n, 1}\left\|z_{n, 1}^{\prime}-q\right\|+\cdots+b_{n, m}\left\|z_{n, m}^{\prime}-q\right\| \\
& \leq b_{n, 0}\left\|x_{n}-q\right\|+b_{n, 1} \operatorname{dist}\left(z_{n, 1}^{\prime}, T_{1} q\right)+\cdots+b_{n, m} \operatorname{dist}\left(z_{n, m}^{\prime}, T_{m} q\right) \\
& \leq b_{n, 0}\left\|x_{n}-q\right\|+b_{n, 1} H\left(T_{1} w_{n}, T_{1} q\right)+\cdots+b_{n, m} H\left(T_{m} w_{n}, T_{m} q\right) \\
& \leq b_{n, 0}\left\|x_{n}-q\right\|+b_{n, 1}\left\|w_{n}-q\right\|+\cdots+b_{n, m}\left\|w_{n}-q\right\| \\
& \leq b_{n, 0}\left\|x_{n}-q\right\|+b_{n, 1}\left\|x_{n}-q\right\|+\cdots+b_{n, m}\left\|x_{n}-q\right\| \leq\left\|x_{n}-q\right\| .
\end{aligned}
$$

Therefore $q \in C_{n}$, which implies that

$$
\mathcal{F}=\bigcap_{i=1}^{m} F\left(T_{i}\right) \cap E P\left(\Phi_{1}\right) \cap E P\left(\Phi_{2}\right) \subset C_{n}, \quad \text { for all } n \geq 0 \text {. }
$$

We observe that $C_{n}$ is closed and convex (see [32]). Now we show that $\lim _{n \rightarrow \infty}\left\|x_{n}-x_{0}\right\|$ exists. By Lemma 2.6 we have $\mathcal{F}$ is closed and convex. Put $w=P_{\mathcal{F}} x_{0}$. From $x_{n}=P_{C_{n}} x_{0}$ and $x_{n+1} \in C_{n+1} \subset C_{n}$ we have

$$
\left\|x_{n}-x_{0}\right\| \leq\left\|x_{n+1}-x_{0}\right\|
$$

Also from $w \in \mathcal{F} \subset C_{n}$ and $x_{n}=P_{C_{n}} x_{0}$ for all $n \geq 0$, we get that

$$
\left\|x_{n}-x_{0}\right\| \leq\left\|w-x_{0}\right\|
$$

It follows that the sequence $\left\{x_{n}\right\}$ is bounded and nondecreasing. Hence the limit $\lim _{n \rightarrow \infty}\left\|x_{n}-x_{0}\right\|$ exists. We show that $\lim _{n \rightarrow \infty} x_{n}=u \in C$. For $k>n$ we have $x_{k}=P_{C_{k}} x_{0} \in$ $C_{k} \subset C_{n}$. Now by applying Lemma 2.3 we have

$$
\left\|x_{k}-x_{n}\right\|^{2} \leq\left\|x_{k}-x_{0}\right\|^{2}-\left\|x_{n}-x_{0}\right\|^{2} .
$$

Since $\lim _{n \rightarrow \infty}\left\|x_{n}-x_{0}\right\|$ exists, it follows that $\left\{x_{n}\right\}$ is a Cauchy sequence, and hence there exists $u \in C$ such that $\lim _{n \rightarrow \infty} x_{n}=u$. Putting $k=n+1$, in the above inequality we have

$$
\lim _{n \rightarrow \infty}\left\|x_{n+1}-x_{n}\right\|=0
$$

From $x_{n+1} \in C_{n+1}$, we have

$$
\left\|y_{n}-x_{n+1}\right\| \leq\left\|x_{n}-x_{n+1}\right\|
$$

so that $\lim _{n \rightarrow \infty}\left\|y_{n}-x_{n+1}\right\|=0$. This implies that $\lim _{n \rightarrow \infty} y_{n}=u$. Take $q \in \mathcal{F}$. By Lemma 2.1, for each $1 \leq i \leq m$, we have

$$
\begin{aligned}
\left\|w_{n}-q\right\|^{2} & =\left\|a_{n, 0} v_{n}+a_{n, 1} z_{n, 1}+\cdots+a_{n, m} z_{n, m}-q\right\|^{2} \\
& \leq a_{n, 0}\left\|v_{n}-q\right\|^{2}+a_{n, 1}\left\|z_{n, 1}-q\right\|^{2}
\end{aligned}
$$




$$
\begin{aligned}
& +\cdots+a_{n, m}\left\|z_{n, m}-q\right\|^{2}-a_{n, i} a_{n, o}\left\|v_{n}-z_{n, i}\right\|^{2} \\
\leq & a_{n, 0}\left\|v_{n}-q\right\|^{2}+a_{n, 1} \operatorname{dist}\left(z_{n, 1}, T_{1} q\right)^{2} \\
& +\cdots+a_{n, m} \operatorname{dist}\left(z_{n, m}, T_{m} q\right)^{2}-a_{n, i} a_{n, o}\left\|v_{n}-z_{n, i}\right\|^{2} \\
\leq & a_{n, 0}\left\|v_{n}-q\right\|^{2}+a_{n, 1} H\left(T_{1} v_{n}, T_{1} q\right)^{2} \\
& +\cdots+a_{n, m} H\left(T_{m} v_{n}, T_{m} q\right)^{2}-a_{n, i} a_{n, o}\left\|v_{n}-z_{n, i}\right\|^{2} \\
\leq & a_{n, 0}\left\|v_{n}-q\right\|^{2}+a_{n, 1}\left\|v_{n}-q\right\|^{2} \\
& +\cdots+a_{n, m}\left\|v_{n}-q\right\|^{2}-a_{n, i} a_{n, o}\left\|v_{n}-z_{n, i}\right\|^{2} \\
\leq & \left\|v_{n}-q\right\|^{2}-a_{n, i} a_{n, o}\left\|v_{n}-z_{n, i}\right\|^{2},
\end{aligned}
$$

and also

$$
\begin{aligned}
\left\|y_{n}-q\right\|^{2}= & \left\|b_{n, 0} v_{n}+b_{n, 1} z_{n, 1}^{\prime}+\cdots+b_{n, m} z_{n, m}^{\prime}-q\right\|^{2} \\
\leq & b_{n, 0}\left\|v_{n}-q\right\|^{2}+b_{n, 1}\left\|z_{n, 1}^{\prime}-q\right\|^{2}+\cdots+b_{n, m}\left\|z_{n, m}^{\prime}-q\right\|^{2} \\
\leq & b_{n, 0}\left\|v_{n}-q\right\|^{2}+b_{n, 1} \operatorname{dist}\left(z_{n, 1}^{\prime}, T_{1} q\right)^{2}+\cdots+b_{n, m} \operatorname{dist}\left(z_{n, m}^{\prime}, T_{m} q\right)^{2} \\
\leq & b_{n, 0}\left\|v_{n}-q\right\|^{2}+b_{n, 1} H\left(T_{1} w_{n}, T_{1} q\right)^{2}+\cdots+b_{n, m} H\left(T_{m} w_{n}, T_{m} q\right)^{2} \\
\leq & b_{n, 0}\left\|v_{n}-q\right\|^{2}+b_{n, 1}\left\|w_{n}-q\right\|^{2}+\cdots+b_{n, m}\left\|w_{n}-q\right\|^{2} \\
\leq & b_{n, 0}\left\|v_{n}-q\right\|^{2}+b_{n, 1}\left\|v_{n}-q\right\|^{2}+\cdots+b_{n, m}\left\|v_{n}-q\right\|^{2} \\
& \quad-b_{n, i} a_{n, i} a_{n, o}\left\|v_{n}-z_{n, i}\right\|^{2} \\
\leq & \left\|x_{n}-q\right\|^{2}-b_{n, i} a_{n, i} a_{n, o}\left\|v_{n}-z_{n, i}\right\|^{2} .
\end{aligned}
$$

So we have that

$$
\begin{aligned}
a^{2} b\left\|v_{n}-z_{n, i}\right\|^{2} & \leq b_{n, i} a_{n, i} a_{n, o}\left\|v_{n}-z_{n, i}\right\|^{2} \\
& \leq\left\|v_{n}-q\right\|^{2}-\left\|y_{n}-q\right\|^{2} \\
& \leq\left\|x_{n}-q\right\|^{2}-\left\|y_{n}-q\right\|^{2},
\end{aligned}
$$

which implies that

$$
\lim _{n \rightarrow \infty}\left\|v_{n}-z_{n, i}\right\|=0, \quad \text { for } i=1,2, \ldots, m \text {. }
$$

Hence

$$
\lim _{n \rightarrow \infty} \operatorname{dist}\left(v_{n}, T_{i} v_{n}\right) \leq \lim _{n \rightarrow \infty}\left\|v_{n}-z_{n, i}\right\|=0 \quad(i=1,2, \ldots, m) .
$$

As above $u_{n}=T_{r_{n}}^{\Phi_{1}} x_{n}$ so that

$$
\begin{aligned}
\left\|u_{n}-q\right\|^{2} & =\left\|T_{r_{n}}^{\Phi_{1}} x_{n}-T_{r_{n}}^{\Phi_{1}} q\right\|^{2} \\
& \leq\left\langle T_{r_{n}}^{\Phi_{1}} x_{n}-T_{r_{n}}^{\Phi_{1}} q, x_{n}-q\right\rangle
\end{aligned}
$$




$$
\begin{aligned}
& =\left\langle u_{n}-q, x_{n}-q\right\rangle \\
& =\frac{1}{2}\left(\left\|u_{n}-q\right\|^{2}+\left\|x_{n}-q\right\|^{2}-\left\|x_{n}-u_{n}\right\|^{2}\right)
\end{aligned}
$$

and hence

$$
\left\|u_{n}-q\right\|^{2} \leq\left\|x_{n}-q\right\|^{2}-\left\|x_{n}-u_{n}\right\|^{2} .
$$

And also by $u_{n}^{\prime}=T_{s_{n}}^{\Phi_{2}} x_{n}$ we have

$$
\begin{aligned}
\left\|u_{n}^{\prime}-q\right\|^{2} & =\left\|T_{s_{n}}^{\Phi_{2}} x_{n}-T_{s_{n}}^{\Phi_{2}} q\right\|^{2} \\
& \leq\left\langle T_{s_{n}}^{\Phi_{2}} x_{n}-T_{s_{n}}^{\Phi_{2}} q, x_{n}-q\right\rangle \\
& =\left\langle u_{n}^{\prime}-q, x_{n}-q\right\rangle \\
& =\frac{1}{2}\left(\left\|u_{n}^{\prime}-q\right\|^{2}+\left\|x_{n}-q\right\|^{2}-\left\|x_{n}-u_{n}^{\prime}\right\|^{2}\right)
\end{aligned}
$$

and hence

$$
\left\|u_{n}^{\prime}-q\right\|^{2} \leq\left\|x_{n}-q\right\|^{2}-\left\|x_{n}-u_{n}^{\prime}\right\|^{2}
$$

Now we use (2) and (3) to obtain

$$
\begin{aligned}
\left\|v_{n}-q\right\|^{2} & \leq \delta_{n}\left\|u_{n}-q\right\|^{2}+\left(1-\delta_{n}\right)\left\|u_{n}^{\prime}-q\right\|^{2} \\
& \leq\left\|x_{n}-q\right\|^{2}-\delta_{n}\left\|x_{n}-u_{n}\right\|^{2}-\left(1-\delta_{n}\right)\left\|x_{n}-u_{n}^{\prime}\right\|^{2} .
\end{aligned}
$$

It follows from (1) and the last inequality that

$$
\left\|y_{n}-q\right\|^{2} \leq\left\|v_{n}-q\right\|^{2} \leq\left\|x_{n}-q\right\|^{2}-\delta_{n}\left\|x_{n}-u_{n}\right\|^{2}-\left(1-\delta_{n}\right)\left\|x_{n}-u_{n}^{\prime}\right\|^{2} .
$$

So we have

$$
a\left\|x_{n}-u_{n}\right\|^{2} \leq \delta_{n}\left\|x_{n}-u_{n}\right\|^{2} \leq\left\|x_{n}-q\right\|^{2}-\left\|y_{n}-q\right\|^{2},
$$

and

$$
(1-b)\left\|x_{n}-u_{n}^{\prime}\right\|^{2} \leq\left(1-\delta_{n}\right)\left\|x_{n}-u_{n}^{\prime}\right\|^{2} \leq\left\|x_{n}-q\right\|^{2}-\left\|y_{n}-q\right\|^{2} .
$$

Since $\lim _{n \rightarrow \infty} x_{n}=\lim _{n \rightarrow \infty} y_{n}=u$, we obtain that

$$
\lim _{n \rightarrow \infty}\left\|u_{n}-x_{n}\right\|=\lim _{n \rightarrow \infty}\left\|u_{n}^{\prime}-x_{n}\right\|=0,
$$

which implies that

$$
\lim _{n \rightarrow \infty}\left\|v_{n}-x_{n}\right\|=0
$$


Since $\lim _{n \rightarrow \infty}\left\|v_{n}-x_{n}\right\|=0$, for $i=1,2, \ldots, m$, we obtain that

$$
\begin{aligned}
\operatorname{dist}\left(x_{n}, T_{i} x_{n}\right) & \leq\left\|x_{n}-v_{n}\right\|+\operatorname{dist}\left(v_{n}, T_{i} v_{n}\right)+H\left(T_{i} v_{n}, T_{i} x_{n}\right) \\
& \leq(\eta+1)\left\|x_{n}-v_{n}\right\|+(\mu+1) \operatorname{dist}\left(v_{n}, T_{i} v_{n}\right) \rightarrow 0 \quad \text { as } n \rightarrow \infty
\end{aligned}
$$

We observe that $u \in \bigcap_{i=1}^{m} F\left(T_{i}\right)$. Indeed,

$$
\begin{aligned}
\operatorname{dist}\left(u, T_{i} u\right) & \leq\left\|u-x_{n}\right\|+\operatorname{dist}\left(x_{n}, T_{i} x_{n}\right)+H\left(T_{i} x_{n}, T_{i} u\right) \\
& \leq(\eta+1)\left\|u-x_{n}\right\|+(\mu+1) \operatorname{dist}\left(x_{n}, T_{i} x_{n}\right) \rightarrow 0 \quad \text { as } n \rightarrow \infty
\end{aligned}
$$

which implies that $u \in \bigcap_{i=1}^{m} F\left(T_{i}\right)$. Let us show that $u \in E P\left(\Phi_{1}\right) \cap E P\left(\Phi_{2}\right)$. From $\lim _{n \rightarrow \infty} x_{n}=u$ and $\lim _{n \rightarrow \infty}\left\|x_{n}-u_{n}\right\|=0$, we have $u_{n} \rightarrow u$ as $n \rightarrow \infty$. Since $u_{n}=T_{r_{n}}^{\Phi_{1}} x_{n}$ we obtain

$$
\Phi_{1}\left(u_{n}, y\right)+\frac{1}{r_{n}}\left\langle y-u_{n}, u_{n}-x_{n}\right\rangle \geq 0 \quad \forall y \in C .
$$

From (A2), we have

$$
\frac{1}{r_{n}}\left\langle y-u_{n}, u_{n}-x_{n}\right\rangle \geq \Phi_{1}\left(y, u_{n}\right),
$$

and hence

$$
\left\langle y-u_{n}, \frac{u_{n}-x_{n}}{r_{n}}\right\rangle \geq \Phi_{1}\left(y, u_{n}\right) .
$$

Since

$$
\frac{u_{n}-x_{n}}{r_{n}} \rightarrow 0
$$

and $u_{n} \rightarrow u$, from (A4) we have

$$
0 \geq \Phi_{1}(y, u), \quad \forall y \in C .
$$

For $t \in(0,1]$ and $y \in C$, let $y_{t}=t y+(1-t) u$. Since $y, u \in C$, and $C$ is convex we have $y_{t} \in C$ and hence $\Phi_{1}\left(y_{t}, u\right) \leq 0$. So, from (A1) and (A4), we have

$$
0=\Phi_{1}\left(y_{t}, y_{t}\right) \leq t \Phi_{1}\left(y_{t}, y\right)+(1-t) \Phi_{1}\left(y_{t}, u\right) \leq t \Phi_{1}\left(y_{t}, y\right)
$$

which gives $\Phi_{1}\left(y_{t}, y\right) \geq 0$. From (A3) we have $0 \leq \Phi_{1}(u, y), \forall y \in C$ and hence $u \in E P\left(\Phi_{1}\right)$. Similarly, we have $u \in E P\left(\Phi_{2}\right)$. Now we show that $u=P_{\mathcal{F}} x_{0}$. Since $x_{n}=P_{C_{n}} x_{0}$, by Lemma 2.2 we have

$$
\left\langle z-x_{n}, x_{0}-x_{n}\right\rangle \leq 0, \quad \forall z \in C_{n} .
$$

Since $u \in \mathcal{F} \subset C_{n}$ we get

$$
\left\langle z-u, x_{0}-u\right\rangle \leq 0, \quad \forall z \in \mathcal{F}
$$

Now by Lemma 2.2 we obtain that $u=P_{\mathcal{F}} x_{0}$. 
By substituting $P_{T_{i}}$ by $T_{i}$ and using a similar argument as in Theorem 3.1, we obtain the following result.

Theorem 3.2 Let $C$ be a nonempty closed convex subset of a real Hilbert space $H, \Phi_{1}$ and $\Phi_{2}$ be two bifunctions of $C \times C$ into $\mathbb{R}$ satisfying (A1)-(A4). Let $T_{i}: C \rightarrow P(C)$, $(i=1,2, \ldots, m)$, be a finite family of multivalued mappings such that each $P_{T_{i}}$ is quasinonexpansive and satisfies the condition $(P)$. Assume further that $\mathcal{F}=\bigcap_{i=1}^{m} F\left(T_{i}\right) \cap$ $\operatorname{EP}\left(\Phi_{1}\right) \cap E P\left(\Phi_{2}\right) \neq \emptyset$. For $C_{0}=C$, let $\left\{x_{n}\right\}$ and $\left\{v_{n}\right\}$ be sequences generated by the following algorithm:

$$
\left\{\begin{array}{l}
x_{0} \in C, \\
u_{n} \in C \text { such that } \Phi_{1}\left(u_{n}, y\right)+\frac{1}{r_{n}}\left\langle y-u_{n}, u_{n}-x_{n}\right\rangle \geq 0 ; \quad \forall y \in C, \\
u_{n}^{\prime} \in C \text { such that } \Phi_{2}\left(u_{n}^{\prime}, y\right)+\frac{1}{s_{n}}\left\langle y-u_{n}^{\prime}, u_{n}^{\prime}-x_{n}\right\rangle \geq 0 ; \quad \forall y \in C, \\
v_{n}=\delta_{n} u_{n}+\left(1-\delta_{n}\right) u_{n}^{\prime}, \\
w_{n}=a_{n, 0} v_{n}+a_{n, 1} z_{n, 1}+\cdots+a_{n, m} z_{n, m}, \\
y_{n}=b_{n, 0} v_{n}+b_{n, 1} z_{n, 1}^{\prime}+\cdots+b_{n, m} z_{n, m}^{\prime}, \\
C_{n+1}=\left\{v \in C_{n}:\left\|y_{n}-v\right\| \leq\left\|x_{n}-v\right\|\right\}, \\
x_{n+1}=P_{C_{n+1}} x_{0}: \quad \forall n \geq 0,
\end{array}\right.
$$

where $z_{n, i} \in P_{T_{i}}\left(v_{n}\right), z_{n, i}^{\prime} \in P_{T_{i}}\left(w_{n}\right)$ for $i=1,2, \ldots$, m. Assume that $\left\{a_{n, j}\right\},\left\{b_{n, j}\right\},\left\{\delta_{n}\right\}$ and $\left\{r_{n}\right\}$, $\left\{s_{n}\right\}$ satisfy the following conditions:

(i) $\left\{a_{n, j}\right\},\left\{b_{n, j}\right\},\left\{\delta_{n}\right\} \subset[a, b] \subset(0,1)(j=0,1,2, \ldots, m)$,

(ii) $\left\{r_{n}\right\},\left\{s_{n}\right\} \subset(0, \infty)$, and $\liminf _{n \rightarrow \infty} r_{n}>0$ and $\liminf _{n \rightarrow \infty} s_{n}>0$.

Then, the sequences $\left\{x_{n}\right\}$ and $\left\{v_{n}\right\}$ converge strongly to $P_{\mathcal{F}} x_{0}$.

As a result, for single valued mappings we obtain the following theorem.

Theorem 3.3 Let $C$ be a nonempty closed convex subset of a real Hilbert space $H$ and $\Phi_{1}$ and $\Phi_{2}$ be two bifunctions of $C \times C$ into $\mathbb{R}$ satisfying (A1)-(A4). Let $T_{i}: C \rightarrow C$ ( $i=$ $1,2, \ldots, m)$, be a finite family of quasi-nonexpansive mappings, each satisfying the condition (P). Assume further that $\mathcal{F}=\bigcap_{i=1}^{m} F\left(T_{i}\right) \cap E P\left(\Phi_{1}\right) \cap E P\left(\Phi_{2}\right) \neq \emptyset$. For $C_{0}=C$, let $\left\{x_{n}\right\}$ and $\left\{v_{n}\right\}$ be sequences generated by the following algorithm:

$$
\begin{cases}x_{0} \in C, & \\ u_{n} \in C \text { such that } \Phi_{1}\left(u_{n}, y\right)+\frac{1}{r_{n}}\left\langle y-u_{n}, u_{n}-x_{n}\right\rangle \geq 0 ; \quad \forall y \in C, \\ u_{n}^{\prime} \in C \text { such that } \Phi_{2}\left(u_{n}^{\prime}, y\right)+\frac{1}{s_{n}}\left\langle y-u_{n}^{\prime}, u_{n}^{\prime}-x_{n}\right\rangle \geq 0 ; \quad \forall y \in C, \\ v_{n}=\delta_{n} u_{n}+\left(1-\delta_{n}\right) u_{n}^{\prime}, \\ w_{n}=a_{n, 0} v_{n}+a_{n, 1} T_{1} v_{n}+\cdots+a_{n, m} T_{m} v_{n}, \\ y_{n}=b_{n, 0} v_{n}+b_{n, 1} T_{1} w_{n}+\cdots+b_{n, m} T_{m} w_{n}, \\ C_{n+1}=\left\{v \in C_{n}:\left\|y_{n}-v\right\| \leq\left\|x_{n}-v\right\|\right\}, \\ x_{n+1}=P_{C_{n+1}} x_{0}: \quad \forall n \geq 0 .\end{cases}
$$

Assume that $\left\{a_{n, j}\right\},\left\{b_{n, j}\right\},\left\{\delta_{n}\right\}$ and $\left\{r_{n}\right\},\left\{s_{n}\right\}$ satisfy the following conditions:

(i) $\left\{a_{n, j}\right\},\left\{b_{n, j}\right\},\left\{\delta_{n}\right\} \subset[a, b] \subset(0,1)(j=0,1,2, \ldots, m)$, 
(ii) $\left\{r_{n}\right\},\left\{s_{n}\right\} \subset(0, \infty)$, and $\liminf _{n \rightarrow \infty} r_{n}>0$ and $\liminf _{n \rightarrow \infty} s_{n}>0$.

Then, the sequences $\left\{x_{n}\right\}$ and $\left\{v_{n}\right\}$ converge strongly to $P_{\mathcal{F}} x_{0}$.

Theorem 3.4 Let $C$ be a nonempty closed convex subset of a real Hilbert space H. Let $T_{i}: C \rightarrow P(C)(i=1,2, \ldots, m)$, be a finite family of multivalued mappings such that $P_{T_{i}}$ is quasi-nonexpansive and satisfies the condition $(P)$. Assume further that $\mathcal{F}=\bigcap_{i=1}^{m} F\left(T_{i}\right) \neq \emptyset$. For $C_{0}=C$, let $\left\{x_{n}\right\}$ be the sequence generated by the following algorithm:

$$
\left\{\begin{array}{l}
x_{0} \in C, \\
w_{n}=a_{n, 0} x_{n}+a_{n, 1} z_{n, 1}+\cdots+a_{n, m} z_{n, m}, \\
y_{n}=b_{n, 0} x_{n}+b_{n, 1} z_{n, 1}^{\prime}+\cdots+b_{n, m} z_{n, m}^{\prime}, \\
C_{n+1}=\left\{v \in C_{n}:\left\|y_{n}-v\right\| \leq\left\|x_{n}-v\right\|\right\}, \\
x_{n+1}=P_{C_{n+1}} x_{0}: \quad \forall n \geq 0,
\end{array}\right.
$$

where $z_{n, i} \in P_{T_{i}}\left(x_{n}\right), z_{n, i}^{\prime} \in P_{T_{i}}\left(w_{n}\right)$ for $i=1,2, \ldots$, m. Assume that $\left\{a_{n, j}\right\},\left\{b_{n, j}\right\} \subset[a, b] \subset(0,1)$ $(j=0,1,2, \ldots, m)$, Then, the sequence $\left\{x_{n}\right\}$ converges strongly to $P_{\mathcal{F}} x_{0}$.

Proof Putting $\Phi_{1}(x, y)=\Phi_{2}(x, y)=0$ for all $x, y \in C$ and $r_{n}=s_{n}=1$ in Theorem 3.2, we have $u_{n}=u_{n}^{\prime}=x_{n}$ and hence $v_{n}=x_{n}$. Now, the desired conclusion follows directly from Theorem 3.2.

Now, we supply an example to illustrate the main result of this paper.

Example 3.5 We consider the nonempty closed convex subset $C=[0,5]$ of the Hilbert space $\mathbb{R}$. Define two mappings $T_{1}$ and $T_{2}$ on $C$ as follows:

$$
T_{1}(x)=\left[\frac{x}{6}, \frac{x}{2}\right], \quad T_{2}(x)= \begin{cases}{\left[0, \frac{x}{5}\right],} & x \neq 5 \\ \{1\}, & x=5 .\end{cases}
$$

We note that $T_{1}$ and $T_{2}$ are quasi-nonexpansive mappings satisfying the condition (P), (for details, see [2]). Also we define two bifunctions $\Phi_{1}$ and $\Phi_{2}$ as follows:

$$
\left\{\begin{array} { l } 
{ \Phi _ { 1 } : C \times C \rightarrow \mathbb { R } , } \\
{ \Phi _ { 1 } ( x , y ) = y - x , }
\end{array} \quad \left\{\begin{array}{l}
\Phi_{2}: C \times C \rightarrow \mathbb{R}, \\
\Phi_{2}(x, y)=y^{2}+x y-2 x^{2} .
\end{array}\right.\right.
$$

It is easy to see that $\Phi_{1}$ and $\Phi_{2}$ satisfy the conditions (A1)-(A4). If we put $r_{n}=5$ and $s_{n}=1$, then $u_{n}=T_{r_{n}}^{\Phi_{1}} x_{n}=0$ and $u_{n}^{\prime}=T_{s_{n}}^{\Phi_{1}} x_{n}=\frac{x_{n}}{3 s_{n}+1}=\frac{x_{n}}{4}$ (for details, see [26]). Put $a_{n, i}=b_{n, i}=\frac{1}{3}$ for $i=0,1,2$ and $\delta_{n}=\frac{1}{2}$. For any arbitrary $x_{0} \in C$ we have

$$
C_{1}=\left\{v \in C:\left|y_{0}-v\right| \leq\left|x_{0}-v\right|\right\}=\left[0, \frac{x_{0}+y_{0}}{2}\right] \text {. }
$$

Since $\frac{x_{0}+y_{0}}{2} \leq x_{0}$, we obtain that

$$
x_{1}=P_{C_{1}} x_{0}=\frac{x_{0}+y_{0}}{2} .
$$


By continuing this process we obtain

$$
C_{n+1}=\left\{v \in C_{n}:\left|y_{n}-v\right| \leq\left|x_{n}-v\right|\right\}=\left[0, \frac{x_{n}+y_{n}}{2}\right] \text {, }
$$

and hence

$$
x_{n+1}=P_{C_{n+1}} x_{0}=\frac{x_{n}+y_{n}}{2} .
$$

Now, we have the following algorithm:

$$
\left\{\begin{array}{l}
x_{0} \in C, \\
v_{n}=\frac{x_{n}}{8}, \\
z_{n, i} \in T_{i}\left(v_{n}\right), \quad i=1,2, \\
w_{n}=\frac{1}{3} v_{n}+\frac{1}{3} z_{n, 1}+\frac{1}{3} z_{n, 2}, \\
z_{n, i}^{\prime} \in T_{i} w_{n}, \quad i=1,2, \\
y_{n}=\frac{1}{3} v_{n}+\frac{1}{3} z_{n, 1}^{\prime}+\frac{1}{3} z_{n, 2}^{\prime} \\
x_{n+1}=\frac{x_{n}+y_{n}}{2}: \quad \forall n \geq 0 .
\end{array}\right.
$$

Putting $z_{n, 1}=z_{n, 2}=\frac{v_{n}}{6}$ and $z_{n, 1}^{\prime}=z_{n, 2}^{\prime}=\frac{w_{n}}{6}$ we get that

$$
x_{n+1}=\frac{679}{1,296} x_{n}=\left(\frac{679}{1,296}\right)^{n+1} x_{0}, \quad \forall n \geq 0 .
$$

We observe that for an arbitrary $x_{0} \in C, x_{n}$ is convergent to zero. We note that $\mathcal{F}=F\left(T_{1}\right) \cap$ $F\left(T_{2}\right) \cap E P\left(\Phi_{1}\right) \cap E P\left(\Phi_{2}\right)=\{0\}$.

Remark 3.6 Since every nonexpansive mapping is quasi-nonexpansive and satisfies the condition (P), our results hold for nonexpansive mappings.

Remark 3.7 Our results generalize the results of Tada and Takahashi [15], of a nonexpansive single valued mapping to a finite family of generalized nonexpansive multivalued mappings.

\section{Competing interests}

The authors declare that they have no competing interests.

Authors' contributions

All the authors contributed equally to the writing of the present article. And they also read and approved the final manuscript.

\section{Author details}

${ }^{1}$ Department of Mathematics, Imam Khomeini International University, Qazvin, 34149, Iran. ${ }^{2}$ Young Researchers Club, Babol Branch, Islamic Azad University, Babol, Iran.

\section{Acknowledgements}

Research of the first author was supported in part by a grant from Imam Khomeini International University, under the grant number 751164-91

Received: 18 December 2011 Accepted: 6 July 2012 Published: 23 July 2012 


\section{References}

1. Garcia-Falset, J, Llorens-Fuster, E, Suzuki, T: Fixed point theory for a class of generalized nonexpansive mappings. J. Math. Anal. Appl. 375, 185-195 (2011)

2. Abkar, A, Eslamian, M: Common fixed point results in CAT(0) spaces. Nonlinear Anal. 74, 1835-1840 (2011)

3. Shahzad, N, Zegeye, H: Strong convergence results for nonself multimaps in Banach spaces. Proc. Am. Math. Soc. 136 539-548 (2008)

4. Song, Y, Wang, H: Convergence of iterative algorithms for multivalued mappings in Banach spaces. Nonlinear Anal. 70, 1547-1556 (2009)

5. Shahzad, N, Zegeye, H: On Mann and Ishikawa iteration schemes for multivalued maps in Banach space. Nonlinear Anal. 71, 838-844 (2009)

6. Eslamian, M, Abkar, A: One-step iterative process for a finite family of multivalued mappings. Math. Comput. Model 54, 105-111 (2011)

7. Nanan, N, Dhompongsa, S: A common fixed point theorem for a commuting family of nonexpansive mappings one of which is multivalued. Fixed Point Theory Appl. 2011, 54 (2011)

8. Abkar, A, Eslamian, M: Convergence theorems for a finite family of generalized nonexpansive multivalued mappings in CAT(0) spaces. Nonlinear Anal. 75, 1895-1903 (2012)

9. Fan, K: A minimax inequality and applications. In: O Shisha (ed.) Inequalities III, pp. 103-113. Academic Press, New York (1972)

10. Brezis, H, Nirembero, L, Stampacchia, G: A remark on Ky Fan's minimax principle. Boll. Unione Mat. Ital. 6, 293-300 (1972)

11. Blum, E, Oettli, W: From optimization and variational inequalities to equilibrium problems. Math. Stud. 63, 123-145 (1994)

12. Flam, SD, Antipin, AS: Equilibrium programming using proximal-link algorithms. Math. Program. 78, $29-41$ (1997)

13. Moudafi, A, Thera, M: Proximal and Dynamical Approaches to Equilibrium Problems. Lecture Notes in Economics and Mathematical Systems, vol. 477, Springer, New York, 187-201 (1999)

14. Combettes, PL, Hirstoaga, SA: Equilibrium programming in Hilbert spaces. J. Nonlinear Convex Anal. 6, 117-136 (2005)

15. Tada, A, Takahashi, W: Weak and strong convergence theorems for a nonexpansive mapping and an equilibrium problem. J. Optim. Theory Appl. 133, 359-370 (2007)

16. Takahashi, S, Takahashi, W: Viscosity approximation methods for equilibrium problems and fixed point problems in Hilbert spaces. J. Math. Anal. Appl. 331, 506-515 (2007)

17. Moudafi, A: Weak convergence theorems for nonexpansive mappings and equilibrium problems. J. Nonlinear Convex Anal. 9, 37-43 (2008)

18. Chang, SS, Lee, HWJ, Kim, JK: A new method for solving equilibrium problem fixed point problem and variational inequality problem with application to optimization. Nonlinear Anal. 70, 3307-3319 (2009)

19. Cho, YJ, Qin, X, Kang, J: Convergence theorems based on hybrid methods for generalized equilibrium problems and fixed point problems. Nonlinear Anal. 71, 4203-4214 (2009)

20. Ceng, LC, Al-Homidan, S, Ansari, QH, Yao, JC: An iterative scheme for equilibrium problems and fixed point problems of strict pseudo-contraction mappings. J. Comput. Appl. Math. 223, 967-974 (2009)

21. Jaiboon, C, Kumam, P: Strong convergence for generalized equilibrium problems, fixed point problems and relaxed cocoercive variational inequalities. J. Inequal. Appl. 2010, Article ID 728028 (2010). doi:10.1155/2010/728028

22. Jung, JS: A general composite iterative method for generalized mixed equilibrium problems, variational inequality problems and optimization problems. J. Inequal. Appl. 2011, 51 (2011)

23. Ceng, LC, Ansari, QH, Yao, JC: Viscosity approximation methods for generalized equilibrium problems and fixed point problems. J. Glob. Optim. 43, 487-502 (2009)

24. Zeng, LC, Ansari, QH, Shyu, DS, Yao, JC: Strong and weak convergence theorems for common solutions of generalized equilibrium problems and zeros of maximal monotone operators. Fixed Point Theory Appl. 2010, Article ID 590278 (2010)

25. Ceng, LC, Ansari, QH, Yao, JC: Hybrid pseudoviscosity approximation schemes for equilibrium problems and fixed point problems of infinitely many nonexpansive mappings. Nonlinear Anal. Hybrid Syst. 4, 743-754 (2010)

26. Singthong, U, Suantai, S: Equilibrium problems and fixed point problems for nonspreading-type mappings in Hilbert space. Int. J. Nonlinear Anal. Appl. 2, 51-61 (2011)

27. Ceng, LC, Ansari, QH, Yao, JC: Hybrid proximal-type and hybrid shrinking projection algorithms for equilibrium problems, maximal monotone operators and relatively nonexpansive mappings. Numer. Funct. Anal. Optim. 31, 763-797 (2010)

28. Zeng, LC, Al-Homidan, S, Ansari, QH: Hybrid proximal-type algorithms for generalized equilibrium problems, maximal monotone operators and relatively nonexpansive mappings, Fixed Point Theory Appl. 2011, Article ID 973028 (2011)

29. Zeng, LC, Ansari, QH, Schaible, S, Yao, JC: Iterative methods for generalized equilibrium problems, systems of general generalized equilibrium problems and fixed point problems for nonexpansive mappings in Hilbert spaces. Fixed Point Theory 12, 293-308 (2011)

30. Nakajo, K, Takahashi, W: Strong convergence theorems for nonexpansive mappings and nonexpansive semigroups. J. Math. Anal. Appl. 279, 372-379 (2003)

31. Dhompongsa, S, Kaewkhao, A, Panyanak, B: On Kirks strong convergence theorem for multivalued nonexpansive mappings on CAT(0) spaces. Nonlinear Anal. 75, 459-468 (2012)

32. Matinez-Yanes, C, Xu, HK: Strong convergence of the CQ method for fixed point processes. Nonlinear Anal. 64 2400-2411 (2006)

doi:10.1186/1029-242X-2012-164

Cite this article as: Abkar and Eslamian: Strong convergence theorems for equilibrium problems and fixed point problem of multivalued nonexpansive mappings via hybrid projection method. Journal of Inequalities and Applications 2012 2012:164 